\title{
The central role of gut microbiota in drug metabolism and personalized medicine
}

\author{
Gianni Colotti ${ }^{*}, 1$ \& Teresa Rinaldi ${ }^{* *, 2}$ \\ ${ }^{1}$ IBPM-CNR Institute of Molecular Biology \& Pathology, Italian National Research Council, 00185, Rome, Italy \\ ${ }^{2}$ Department of Biology \& Biotechnology, Sapienza University of Rome, 00185, Rome, Italy \\ *Authors for correspondence: gianni.colotti@cnr.it \\ **Authors for correspondence: teresa.rinaldi@uniroma1.it
}

“drugs can profoundly affect microbial gene expression while, on the other hand, microbiota can transform drugs, affecting their bioavailability, efficacy and toxicity"

First draft submitted: 22 January 2020; Accepted for publication: 27 March 2020; Published online: 29 May 2020

Keywords: diseases $\bullet$ drug efficacy and toxicity $\bullet$ drug metabolism • dysbiosis $\bullet$ gut $\bullet$ microbiota $\bullet$ personalized medicine

The gut microbiota is now considered as a symbiotic organ playing an important role in human health and disease development [1] and has been recently recognized as a modulator of drug metabolism and toxicity. Here, we briefly discuss new findings describing how the gut microbiota is now considered to be a central player in drug metabolism and personalized medicine.

\section{Microbiota biology}

The gut microbiota is essential for the digestion of many nutrients and for the synthesis, modification and excretion of metabolites such as lipids, amino acids, bile acids, vitamins and short-chain fatty acids. The microbiota prevents pathogenic bacteria from colonization within the body by competing for their nutrients and by producing peptides and toxins inhibiting their growth. It preserves the integrity of the intestinal epithelium and maintains an optimal local $\mathrm{pH}$ [2].

At early stages, the microbiota produces signals promoting healthy microbial colonization and the development of a functional immune system [3]. It maintains, throughout the lifetime of the individual, the homeostasis of the host-microbiota environment by the production of a set of molecules and by activating cells that promote both the innate and adaptive immunity through the establishment of a complex network of regulatory pathways (e.g., suppression of inflammatory responses).

\section{Microbiota in health \& diseases}

The microbiota of a healthy individual is relatively stable, but gut microbial dynamics are influenced by lifestyle and dietary choices [4]. An interesting example is that Japanese people, who commonly consume seaweed in their diet, harbor in their gut the bacterium Bacteroides plebeius. These bacteria have acquired $\beta$-porphyranase genes by horizontal transfer from a marine bacteroidetes capable of digesting complex carbohydrates [5]. Diet and antibiotic use alters the microbiota, mostly decreasing its diversity, especially in elder people. Western populations, using high amounts of antibiotics and consuming low-fiber diets, harbor in their guts reduced amounts of beneficial bacteria [6].

Reduction in microbiome diversity or dysbiosis can lead to compositional alteration at various taxonomic levels that have been associated with inflammatory bowel disease, Crohn's disease, asthma, rheumatoid arthritis, multiple sclerosis, obesity, metabolic syndrome, cardiovascular diseases, cancer, neurodegenerative and neuropsychiatric diseases.

The gut microbiota has also been shown to modulate physiological processes such as neuroinflammation, serotonin turnover, myelination and the secretion of the prosocial hormone oxytocin, suggesting that the gut microbiota may even have an influence on social behavior [7]. 


\section{Drugs versus microbiota}

\section{Microbiota alteration upon drug administration}

The use of medications can have an impact on the composition of the microbiota as well as the expression of their genes. Maier et al. [8]. recently showed that about $24 \%$ of 1000 drugs tested inhibit at least one bacterial strain of the gut microbiota in vitro. Commonly used drugs, such as antipsychotics, proton pump inhibitors, hormonal therapy and anticancer drugs, have important effects on the microbiota and should not be underestimated $[9,10]$. As an example, metformin, used for the treatment of type 2 diabetes, has a dose-dependent antimicrobial effect, while promoting the growth of bacteria such as Akkermansia muciniphila and Clostridium cocleatum as well as improve lipopolysaccharide biosynthesis [11,12]. In addition, the chronic opioid usage in severe pain management can have an impact on gut motility and is associated with altered gut microbiota [13,14].

\section{Microbiota versus drugs}

\section{Microbiota shapes drug metabolism, efficacy \& toxicity}

The microbiota can perform a series of metabolizing reactions that complement those carried out by the human host; metabolism of xenobiotic compounds (including drugs) is therefore the product of both host-dependent liver and intestinal mucosa-based reactions and of those carried out by gut microbiota [15].

Drug metabolism can be affected by the microbiota in multiple ways. When drugs with low permeability reach the gut, they can be directly metabolized by bacterial enzymes affecting their absorption and bioavailability; alternatively, drugs can be detoxified by the liver and undergo biliary excretion (parenterally administered drugs also reach the liver via the splanchnic circulation); however, liver-detoxified xenobiotics may be reactivated by gut microbiota.

The gut microbiota either contributes to drug degradation or to drug activation or modulate host enzyme activities that metabolize the drugs: many drugs are modified by these mechanisms.

Many chemical reactions that shape drug metabolism are contributed by the microbiota. Hydrolytic and reductive reactions are the main enzymatic activities of gut microbiota drug metabolism, because of the low oxygen concentration in the gut microenvironment [16]. These involve bacterial $\beta$-glucosidases, $\beta$-glucuronidases, aryl sulfatases, azoreductases, nitroreductases and other enzymatic activities, with important roles in xenobiotic metabolism; in addition, chemical reactions including acetylation, deamination, dehydroxylation, decarboxylation, demethylation, deconjugation and proteolysis also impact drug metabolism [16,17]. Examples of drug activation are: the reduction activity on 5-aminosalicylic acid prodrugs as sulfasalazine, olsalazine, balsalazide or ipsalazide, which is carried out by nitro- and azo-reductase enzymes from the genera Clostridia and Eubacteria; reduction of the azo bond of antibacterial drug prontosil to yield triaminobenzene and the bacteriostatic sulfanilamide; the reduction of the $\mathrm{N}$-oxide prodrug loperamideoxide to loperamide $[18,19]$. Drug bioavailability can be affected by gut microbiota: the cardiac glycoside digoxin is reduced to metabolites such as dihydrodigoxin and thereby inactivated by reducing strains of Eggerthella lenta [20].

The deamination of 5-fluorocytosine to 5-fluorouracil by the action of the gut microbiota is responsible, at least in part, for 5 -fluorocytosine chemotherapy toxicity [21]. Drug reactivation by gut bacterial $\beta$-glucuronidases is responsible for the toxicity of irinotecan, a camptotecin-like prodrug used intravenously for the treatment of colorectal cancer: human carboxylases convert irinotecan into the bioactive SN-38 compound which is subsequently inactivated in the liver and transported into the intestine through the biliary route; there, it is converted again into the active $\mathrm{SN}-38$ compound by bacterial $\beta$-glucuronidases, thereby provoking epithelial cell damage, increasing diarrhea and impairing treatment efficacy [22].

Gut bacteria, such as Clostridiales, can positively affect patient responses to anti-PD-1 immunotherapy in melanoma [23] and the presence of the bacterium A. muciniphila in both humans and mice was linked to better responses to anti-PD-1 immunotherapy [24]. In the era of pharmacogenomics, the genetic variability of patients is taken into account to select a personalized therapy with biological drugs, but now microbial molecules or consortia of microorganisms deserve consideration for maximizing the effectiveness of a therapy; we should also be prudent when considering the use of antibiotics in cancer patients as we still have more to learn about how microbiota microbes exert their influence on the immune system.

\section{The microbiota \& personalized medicine}

Based on what we have discussed so far, on the one hand, drugs can profoundly affect microbial gene expression while, on the other hand, microbiota can transform drugs, affecting their bioavailability, efficacy and toxicity [20]: 
for example, about $30 \%$ of type 2 diabetes patients cannot tolerate metformin and $10 \%$ of healthy individuals are poor converters of sulfasalazine, due to variations in microbial phenotype [25].

Recent approaches aim at identifying markers for microbe-drug interaction and microbiome metabolic phenotyping. For example, high risk of irinotecan-dependent toxicity is due to subsets of bacterial $\beta$-glucuronidases which reactivate the irinotecan metabolite SN-38G. The incubation of this metabolite with human fecal samples before drug treatment may suggest the patient's risk of poor drug efficacy and safety [15].

Clinical trials investigating the efficacy of probiotics or of bacterial $\beta$-glucuronidase inhibitors on irinotecandependent toxicity are ongoing, as well as trials on the effect of probiotics on the toxicity of the antineoplastic drugs, VEGF tyrosine kinase inhibitor and of the irreversible EGFR inhibitor dacomitinib.

Microbiota profiling appears important for dosage and discontinuing of treatment with the immunosuppressant tacrolimus, since Clostridiales convert tacrolimus into less active metabolites [26].

In an effort to improve the response to immune therapy, studies on the effect of administration of fecal microbiota transplant together with pembolizumab to patients who have not responded to the monoclonal antibody are currently recruiting patients or ongoing for the treatment of metastatic prostate cancer, gastrointestinal cancers and melanoma. Clinical trials on the efficacy of fecal microbiota transplant are also currently recruiting patients or ongoing for many different pathological conditions, such as neurological diseases, intestinal pathologies, obesity, diabetes, psoriatic arthritis, multiple sclerosis and antibiotic resistance, among others.

Problems, challenges, issues \& solutions for microbiota: looking forward to personalized medicine Although knowledge on the role of microbiota-host interaction has grown exponentially in the last decade, some aspects of this interaction are still poorly understood. It is however well established that a close association between many pathological conditions and dysbiosis exists; a healthy, rich and diverse microbiota is increasingly considered important for maintaining optimal metabolic and immune functions and for preventing disease development. Further recent findings are changing the paradigm of disease treatment, pointing to the possibility of analyzing microbial biomarkers to 'shape' and choose the most suitable and efficacious therapy. It has been shown that the host microbiota can be altered by the use of antibiotics, drugs or bacterial supplementation.

The microbiota is a very complex organ with many challenges remaining before we can completely understand the role it plays in human health and disease. We still have limited information about microbial enzymes, mechanisms, metabolites and pathways, let alone an integrated view of the whole microbiota as an intrinsic regulator of the human health.

Thus, the interdependence of diet components versus nutrient absorption versus fermentation versus bacteria composition and metabolism versus immune system hold many clues for a deeper, multidisciplinary and integrated investigation of this complex system that can lead to a better understanding of how to manipulate, restore and personalize the host-microbiota system achieving effective and reliable personalized medicine.

Everything starts from within.

\section{Financial \& competing interests disclosure}

The authors have no relevant affiliations or financial involvement with any organization or entity with a financial interest in or financial conflict with the subject matter or materials discussed in the manuscript. This includes employment, consultancies, honoraria, stock ownership or options, expert testimony, grants or patents received or pending, or royalties.

No writing assistance was utilized in the production of this manuscript.

\section{References}

1. Backhed F, Ley RE, Sonnenburg JL, Peterson DA, Gordon JI. Host-bacterial mutualism in the human intestine. Science 307(5717), 1915-1920 (2005).

2. Rinninella E, Raoul P, Cintoni M et al. What is the healthy gut microbiota composition? A changing ecosystem across age, environment, diet, and diseases. Microorganisms 7(1), 14 (2019).

3. Belkaid Y, Hand TW. Role of the microbiota in immunity and inflammation. Cell 157(1), 121-141 (2014).

4. Wu GD, Chen J, Hoffmann C et al. Linking long-term dietary patterns with gut microbial enterotypes. Science 334(6025), 105-108 (2011).

5. Hehemann JH, Correc G, Barbeyron T et al. Transfer of carbohydrate-active enzymes from marine bacteria to Japanese gut microbiota. Nature 464, 908-912 (2010). 
6. Sonnenburg ED, Smits SA, Tikhonov M et al. Diet-induced extinctions in the gut microbiota compound over generations. Nature 529, 212-215 (2016).

7. Sherwin E, Bordenstein SR, Quinn JL, Dinan TG, Cryan JF. Microbiota and the social brain. Science 366 (6465) eaar2016 (2019).

8. Maier L, Pruteanu M, Kuhn M et al. Extensive impact of non-antibiotic drugs on human gut bacteria. Nature 555, 623-628 (2018).

9. Clarke G, Sandhu KV, Griffin BT et al. Gut reactions: breaking down xenobiotic-microbiome interactions. Pharmacol. Rev. 71(2), 198-224 (2019).

10. Enright EF, Gahan CG, Joyce SA, Griffin BT. The impact of the gut microbiota on drug metabolism and clinical outcome. Yale J. Biol. Med. 89(3), 375-382 (2016).

11. Lee H, Ko G. Effect of metformin on metabolic improvement and gut microbiota. Appl. Environ Microbiol. 80(19), 5935-5943 (2014).

12. Wu W, Lv L, Shi D et al. Protective effect of akkermansia muciniphila against immune-mediated liver injury in a mouse model. Front. microbiol. 8, 1804 (2017).

13. Banerjee S, Sindberg G Wang F et al. Opioid-induced gut microbial disruption and bile dysregulation leads to gut barrier compromise and sustained systemic inflammation. Mucosal Immunol. 9(6), 1418-1428 (2016).

14. Lee K, Vuong HE, Nusbaum DJ et al. The gut microbiota mediates reward and sensory responses associated with regimen-selective morphine dependence. Neuropsychopharmacology 43(13), 2606-2614 (2018).

15. Guthrie L, Kelly L. Bringing microbiome-drug interaction research into the clinic. EBioMedicine 44, 708-715 (2019).

16. Wilson ID, Nicholson JK. Gut microbiome interactions with drug metabolism, efficacy, and toxicity. Transl. Res. 179, 204-222 (2017).

17. Spanogiannopoulos P, Bess EN, Carmody RN, Turnbaugh PJ. The microbial pharmacists within us: a metagenomic view of xenobiotic metabolism. Nat. Rev. Microbiol. 14, 273-287 (2016).

18. Lavrijsen K, van Dyck D, van Houdt J et al. Reduction of the prodrug loperamide oxide to its active drug loperamide in the gut of rats, dogs, and humans. Drug Metab. Dispos. 23(3), 354-362 (1995).

19. Rafii F, Cerniglia CE. Reduction of azo dyes and nitroaromatic compounds by bacterial enzymes from the human intestinal tract. Environ. Health Perspect. 103 (Suppl. 5), 17-19 (1995).

20. Haiser HJ, Gootenberg DB, Chatman K et al. Predicting and manipulating cardiac drug inactivation by the human gut bacterium Eggerthella lenta. Science 341(6143), 295-298 (2013).

21. Harris BE, Manning BW, Federle TW, Diasio RB. Conversion of 5-fluorocytosine to 5-fluorouracil by human intestinal microflora. Antimicrob. Agents Chemother. 29(1), 44-48 (1986).

22. Stein A, Voigt W, Jordan K. Chemotherapy-induced diarrhea: pathophysiology, frequency and guideline-based management. Ther. Adv. Med. Oncol. 2(1), 51-63 (2010).

23. Gopalakrishnan V, Spencer CN, Nezi L et al. Gut microbiome modulates response to anti-PD-1 immunotherapy in melanoma patients. Science 359(6371), 97-103 (2018).

24. Routy B, Le Chatelier E, Derosa L et al. Gut microbiome influences efficacy of PD-1-based immunotherapy against epithelial tumors. Science 359(6371), 91-97 (2018).

25. Klotz U, Maier K, Fischer C, Heinkel K. Therapeutic efficacy of sulfasalazine and its metabolites in patients with ulcerative colitis and Crohn's disease. N. Engl. J. Med. 303 (26), 1499-1502 (1980).

26. Guo Y, Crnkovic CM, Won KJ et al. Commensal gut bacteria convert the immunosuppressant tacrolimus to less potent metabolites. Drug Metab. Dispos. 47(3), 194-202 (2019). 